\title{
Commentary \\ Are herbal remedies and dietary supplements safe and effective for breast cancer patients?
}

\author{
Aedin Cassidy \\ Hugh Sinclair Human Nutrition Unit, School of Food Biosciences, University of Reading, Reading, UK \\ Corresponding author: Aedin Cassidy (e-mail: cassidyaedin@hotmail.com) \\ Published: 7 October 2003
}

Breast Cancer Res 2003, 5:300-302 (DOI 10.1186/bcr724)

(c) 2003 BioMed Central Ltd (Print ISSN 1465-5411; Online ISSN 1465-542X)

See related Research article: http://breast-cancer-research.com/content/5/6/R170

\begin{abstract}
There remains limited scientific evidence on the efficacy and safety of 'natural' therapies such as herbal remedies and dietary supplements. Nevertheless, breast cancer patients are particularly prone to purchasing such products because of the perception that 'natural' products are less toxic than conventional prescribed medicines. However, the potential for interactions of supplements with current medications, the potential for adverse effects from consumption at high levels, and the lack of disclosure of such treatments by the patient to their doctor are serious public health issues. Robust clinical trials are required to prove the efficacy and lack of adverse effects of such preparations, and communication between patients and doctors must be improved and doctors made more aware that their patients may be seeking advice and treatment from sources outside conventional medicine.
\end{abstract}

Keywords: breast cancer, dietary, herbal, supplements

\section{Introduction}

Many women are keen to use herbal or 'natural' remedies, either to prevent breast cancer or to assist in treating the disease, but most remain unaware of the limited scientific evidence surrounding the efficacy of these remedies, and the potential drug interactions and adverse effects that may result from their consumption. In many instances these women rely on in-house sale staff of health food shops to provide sufficient and accurate advice. However, an article published in this issue of Breast Cancer Research [1] provides data on the heterogeneity of advice given by sales staff and the limited scientific evidence provided to purchasers of natural or herbal remedies. These data are consistent with previous studies [2-4] and highlight important issues regarding the misleading and potentially harmful nature of the advice provided to breast cancer patients by health food shops.

\section{Risk versus benefit of 'natural' products}

In the study published in this issue [1] a number of products were recommended, none of which are currently supported by scientific evidence to confer a health benefit.
Indeed, in many instances the potential for adverse effects of the products were not discussed, or were drug interactions addressed. The study raises significant concerns, particularly following the recent publication of data from the Women's Health Initiative on the risk-benefit profile of hormone replacement therapy (HRT) [5] and the confirmation from the UK based Million Women Study that current and recent use of HRT increases the risk for breast cancer [6]. These data have been widely publicized in the press, and the headlines produced from such studies may lead women to seek dietary or herbal alternatives to drug therapies such as HRT, under the assumption that 'natural' products are safe and effective.

Women are high consumers of alternative or complementary therapies [7], and thus breast cancer patients are particularly prone to purchasing such remedies. Many of these remedies are based on folklore [8] and have not yet been scrutinized to the scientific rigor that is required for approval of drug therapies. In one study [9], conducted in the USA, the prevalence of use of alternative therapies by breast cancer patients was greatest in women with higher 
educational attainment and income. The key public health issues arising from consumption of such remedies relate to the potential for interactions of supplements with current drug therapy [10], the potential adverse effects associated with consumption, particularly at high doses [11], the concurrent use of several alternative therapies, the costs of such therapies, and the lack of disclosure of such treatments by the patient to their doctor [12].

Alarmingly, only half of the breast cancer patients in one USA-based study reported discussing their use of an alternative therapy with their doctor [9]. Doctors must be more aware that their breast cancer patients may be seeking advice and treatment from sources outside conventional medicine. Poor communication between patient and doctor is therefore a potential area for improvement, and doctors treating patients with breast cancer should initiate a dialogue on alternative therapies to ensure that optimal advice is given regarding treatment options.

Medical science continues to comb through nature's array of compounds for potential substances that may successfully prevent or treat breast cancer. To date, however, most of the herbal remedies or alternative 'natural' preparations on sale to breast cancer patients have either not been robustly tested in appropriately controlled clinical studies or lack clinical data to support their use in breast cancer $[13,14]$. Natural extracts are complex mixtures, and stringent regulations on quality assurance, which apply to drug therapies, are not currently enforced. Indeed, there is evidence to suggest wide variability in the quality of available dietary supplements and herbal remedies, one example of which is the poor correlation between actual and reported content of commercially available dietary phytoestrogen/isoflavone supplements [15].

Breast cancer patients are attracted to 'natural' or alternative therapies because of the perception that they are less toxic than conventional prescribed medicines. Although recent reports on the potential adverse effects and drug interactions resulting from consumption of some natural products [16-18] may have raised awareness within the medical community of the need for appropriate clinical trials to address the ratio of risk to benefit for these preparations, this information is unlikely to have filtered through to staff and purchasers from health food shops. In particular, risk assessment for dose and duration of exposure must be addressed to help define the 'efficacious' dose, if indeed there is any. Health food shops are and will probably remain a frequent source for such products and health advice, but to date they remain unregulated and their employees require no formal education in medical or nutritional science. Currently, the alternative therapy market is governed by marketing, with no regulation to ensure the efficacy or safety of the products on sale. In the future, more formal training of such staff on adverse effects, drug interactions and the limitations of the clinical data on the products sold would help consumers to make a more rational choice. From a manufacturing perspective, it would be ideal to introduce more stringent regulations to ensure that a health benefit can only be marketed following the completion and publication of clinical studies to define optimal dose and assure no potential adverse effects or drug interactions. Doctors must be aware of what products their patients are consuming, and instilling the importance of openness between manufacturers of alternative therapies and the medical community is important for future cooperation.

With the increasing availability of medical and nutritional information to consumers from the internet and other sources, doctors must remain up-to-date on the available complementary or alternative medicines their patients are consuming and offer cautious advice on their use. There is currently insufficient scientific evidence available to suggest that alternative therapies are safe and effective for either the prevention or treatment of breast cancer. Because of this lack of efficacy and potential for adverse effects, their use warrants caution, particularly in vulnerable breast cancer patients.

\section{Conclusion}

Breast cancer patients, along with the public in general, remain unaware that most of the herbal remedies and alternative natural preparations available have not been robustly tested in carefully designed clinical studies. Natural extracts are complex mixtures, and stringent regulations on quality assurance, which apply to drug therapies, are not currently enforced. For most of these products, we still do not know whether they work, and studies are required to define whether they are effective and safe and, if so, what dose is required for such an effect. Until such evidencebased data is available, their use merits caution, particularly in vulnerable breast cancer patients.

\section{Competing interests}

None declared.

\section{References}

1. Mills E, Ernst E, Singh R, Ross C, Wilson K: Health food store recommendations: implications for breast cancer patients. Breast Cancer Res 2003, 5:R170-R174.

2. Gotay CC, Dumitriu D: Health Food store recommendations for breast cancer patients. Arch Fam Med 2000, 9:692-699.

3. Stoffer S, Szpunar W, Coleman B, Mallos P: Advice from some health food stores. JAMA 1980, 244:2044-2046.

4. US Food and Drug Administration: Unsubstantiated Claims and Documented Health Hazards in the Dietary Supplements Marketplace. USA: Food and Drug Administration; 1993.

5. Writing Group of the Women's Health Initiative Investigators: Risks and benefits of estrogen plus progestin in healthy postmenopausal women: principle results from the Women's Health Initiative randomized controlled trial. JAMA 2002, 288: 321-333.

6. Million Women Study Collaborators: Breast cancer and hormone replacement therapy in the Million Women Study. Lancet 2003, 362:419-427. 
7. Morris KT, Johnson N, Homer L, Walts D: A comparison of complementary therapy use between breast cancer patients and patients with other primary tumor sites. Am J Surg 2000, 179: 407-411.

8. Ernst E, Cassileth BR: The prevalence of complementary/alternative medicine in cancer: a systematic review. Cancer 1998 , 83:777-782.

9. Lee MM, Lin SS, Wrensch MR, Adler SR, Eisenberg D: Alternative therapies used by women with breast cancer in four ethnic populations. J Natl Cancer Inst 2000, 92:42-47.

10. Spaulding-Albright $\mathrm{N}$ : A review of some herbal and related products commonly used in cancer patients. J Am Diet Assoc 1997, Suppl 2:S208-215.

11. Mathijssen RH, Verweij J, De Bruijn $P$, Loos WJ, Sparreboom A: Effects of St. John's Wort on irinotecan metabolism. J Natl Cancer Inst 2002, 94:1247-1249.

12. Adler SR, Fosket JR: Disclosing complementary and alternative medicine use in the medical encounter: a qualitative study in women with breast cancer. J Fam Pract 1999, 48:453-458.

13. Kaegi E: Unconventional therapies for cancer: 1. Essiac. The Task Force on Alternative Therapies of the Canadian Breast Cancer Research Initiative. CMAJ 1998, 158:897-902.

14. Miller DR, Anderson GT, Stark JJ, Granick JL, Richardson D: Phase I/II trial of the safety and efficacy of shark cartilage in the treatment of advanced cancer. J Clin Oncol 1998, 16: 3649-3655.

15. Setchell KD, Brown NM, Desai P, Zimmer-Nechemias L, Wolfe BE, Brashear WT, Kirschner AS, Cassidy A, Heubi JE: Bioavailability of pure isoflavones in healthy humans and analysis of commercial soy isoflavone supplements. J Nutr 2001, Suppl:1362S-1375S

16. US Food and Drug Administration: Risk of drug interactions with St. John's Wort. JAMA 2000, 283:1679.

17. Izzo AA, Ernst $E$ : Interactions between herbal medicines and prescribed drugs:a systematic review. Drugs 2001, 61:21632175.

18. US Food and Drug Administration: Letter to Health Care Professionals. FDA Issues Consumer Advisory that Kava Products may be Associated with Severe Liver Injury. USA: US Food and Drug Administration, Office of Nutritional Products, Labelling and Dietary Supplements; 2002.

\section{Correspondence}

Aedin Cassidy, Hugh Sinclair Human Nutrition Unit, School of Food Biosciences, University of Reading, Whiteknights, Reading, RG6 6AP, UK. Tel/fax: +44 (0)1234 870498; e-mail: cassidyaedin@hotmail.com 